\title{
Branched-chain amino acid supplementation in adults with cirrhosis and hepatic encephalopathy: a systematic review and meta-analyses
}

\author{
E. L. Metcalfe ${ }^{1,2}$, A. Avenell ${ }^{1}$ and A. Fraser ${ }^{2}$ \\ ${ }^{1}$ Health Services Research Unit, University of Aberdeen, Foresterhill, Aberdeen, AB25 2ZD, UK and ${ }^{2}$ Gastroenterology and \\ Hepatology Unit, Aberdeen Royal Infirmary, Aberdeen, AB25 2ZN, UK
}

Branched-chain amino acid (BCAA) supplementation for the treatment of hepatic encephalopathy (HE) remains controversial. A Cochrane review (last assessed as up-to-date in 2002) compared BCAAs to any other treatment for HE resulting from either acute or chronic liver disease or fulminant hepatic failure, but found no evidence of survival benefit and, in studies of adequate methodological quality, no significant improvement in $\mathrm{HE}^{(1)}$. However, the ESPEN nutritional guidelines from 2009 are at variance with these results, suggesting that patients with more severe HE (i.e. grade III-IV) receive supplementation ${ }^{(2)}$. This systematic review was undertaken to update the evidence for the role of BCAA supplementation in adults with cirrhosis and HE in terms of the effect on encephalopathy, hepatic decompensation, survival, infection, hospital stay and quality of life, and to obtain data on adherence, side-effect profile and any cost/ economic evaluation where available.

A systematic review of randomised controlled trials (RCTs) was undertaken by searching MEDLINE, Embase, the Cochrane Central Register of Controlled Trials and BIOSIS from 1980 to June 2011. No language restrictions were imposed. Hand-searching was performed on reference lists of included trials in addition to conference proceedings for BAPEN, ESPEN, ASPEN, EASL, AASLD and AGA for 2005-2010. The search identified thirteen randomised controlled trials (572 patients in total) which together provided fourteen comparisons of BCAA therapy for $\geq 2$ weeks' duration versus either 'other nutritional supplements' $(n=9)$, 'nil/ placebo' $(n=3)$ or 'other therapy' $(n=2)$ in adult patients with cirrhosis and HE. Data were extracted by two independent assessors. Sufficient data for metaanalyses were only available for the 'other nutritional supplement' comparison on HE, hepatic decompensation, gastrointestinal bleeding, survival and infection. Meta-analysis was performed using Review Manager 5 and the random effects model was used in view of the anticipated variability between trials. No studies included any cost/ economic evaluation. Side-effects, where reported, were mild and gastrointestinal in nature. The limited data available for health-related quality of life, adherence to therapy, ascites and admission to hospital did not suggest any significant difference between therapies.

\begin{tabular}{llc}
\hline Outcome & Risk Ratio & Significance $(P)$ \\
\hline Resolution of hepatic encephalopathy (2 RCT; n 107) & RR 3.9 & $0.6,23.7$ \\
Improvement in hepatic encephalopathy (2 RCT; n 122) & RR 2.6 & 0.14 \\
Worsening of hepatic encephalopathy (3 RCT; n 200) & RR 0.6 & $1.7,3.9$ \\
Gastrointestinal bleeding (2 RCT; n 180) & RR 1.1 & $0.3,1.3$ \\
Survival (2 RCT; n 180) & RR 1.1 & $0.2,7.3$ \\
Infection (2 RCT; n 142) & RR 1.7 & $1.0,1.1$ \\
\hline
\end{tabular}

This systematic review and meta-analyses suggest that while BCAA therapy might lead to a significant improvement in HE compared to 'other nutritional supplements', there is no evidence to support its role in improving overall survival. However, given the small number of trials involved, most of which were of low methodological quality with small numbers of patients, a large multi-centre randomised controlled trial of sound methodological quality, incorporating an economic evaluation, would be justified before a final conclusion is reached.

1. Als-Nielson B et al. (2003) Branched-chain amino acids for hepatic encephalopathy. Cochrane Database of Systematic Reviews Issue 1.

2. Plauth M et al. (2009) Clin Nutr 28, 436-444. 